\title{
Microcollimator for Micrometer-Wide Stripe Irradiation of Cells Using 20-30 keV X Rays
}

\author{
Kristopher Pataky, ${ }^{a}$ Guillermo Villanueva,${ }^{a}$ Andre Liani, ${ }^{b}$ Omar Zgheib,${ }^{b}$ Nathan Jenkins, ${ }^{c}$ Demetrios J. Halazonetis,${ }^{d}$ \\ Thanos D. Halazonetis ${ }^{b, e}$ and Juergen Brugger ${ }^{a, 1}$ \\ ${ }^{a}$ Microsystems Laboratory, Ecole Polytechnique Federale de Lausanne (EPFL), Lausanne, Switzerland; ${ }^{b}$ Department of Molecular Biology, \\ University of Geneva, Geneva, Switzerland; ' Department of Physics, University of Geneva, Geneva, Switzerland; ${ }^{d}$ Department of Orthodontics, \\ University of Athens, Athens, Greece; and e Department of Biochemistry, University of Geneva, Geneva, Switzerland
}

Pataky, K., Villanueva, G., Liani, A., Zgheib, O., Jenkins, N., Halazonetis, D. J., Halazonetis, T. D. and Brugger, J. Microcollimator for Micrometer-Wide Stripe Irradiation of Cells Using 20-30 keV X Rays. Radiat. Res. 172, 252-259 (2009).

The exposure of subnuclear compartments of cells to ionizing radiation is currently not trivial. We describe here a collimator for micrometer-wide stripe irradiation designed to work with conventional high-voltage $\mathrm{X}$-ray tubes and cells cultured on standard glass cover slips. The microcollimator was fabricated by high-precision silicon micromachining and consists of X-ray absorbing chips with grooves of highly controlled depths, between $0.5-10 \mu \mathrm{m}$, along their surfaces. These grooves form $\mathrm{X}$ ray collimating slits when the chips are stacked against each other. The use of this device for radiation biology was examined by irradiating human cells with $\mathrm{X}$ rays having energies between 20-30 keV. After irradiation, p53 binding protein 1 (53BP1), a nuclear protein that is recruited at sites of DNA double-strand breaks, clustered in lines corresponding to the irradiated stripes. $\odot 2009$ by Radiation Research Society

\section{INTRODUCTION}

Ionizing radiation has been widely used in research laboratories to study the effects of DNA double-strand breaks (DSBs) in cells $(1,2)$. The most common sources of radiation for biological experiments are the radioactive isotope ${ }^{137} \mathrm{Cs}$ and high-voltage X-ray tubes, with the latter gaining increasing popularity due to safety and security concerns.

The radiation produced from ${ }^{137} \mathrm{Cs}$ and $\mathrm{X}$-ray tubes can be collimated, allowing part of a biological specimen to be irradiated. It is relatively easy to fabricate collimators made of lead, stainless steel or other metals with slits that have linear dimensions as small as $0.1-1 \mathrm{~mm}(100-1,000 \mu \mathrm{m})$. However, these dimensions are still too large to irradiate parts of a nucleus of a cell, a task that is becoming increasingly useful for the study of the biological responses of cells to DNA DSBs $(3,4)$.

${ }^{1}$ Address for correspondence: BM 3.115/Station 17 EPFL - Microsystems Laboratory 1, Lausanne, 1015, Switzerland; e-mail: juergen. brugger@epfl.ch.
To restrict the linear dimensions of the irradiated area to the micrometer and submicrometer range, microbeams have been developed (5). These microbeams can produce either high- or low-LET radiation. The high-LET radiation microbeams are suitable to study the health effects of exposure to radon and to cosmic rays and employ $\alpha$ particles as well as highly charged, energetic (HZE) particles such as iron and gold to irradiate the biological targets (6-9). The lowLET radiation microbeams were developed to better understand the response of cells to $\mathrm{X}$ rays or $\gamma$ rays and employ ultrasoft $X$ rays, energetic electrons or high-energy protons $(10-13)$.

In microbeams, the dimensions of the irradiated area can be limited to a few micrometers or less using either collimation or focusing. In the case of high-LET radiation microbeams, when a cell is targeted by a single particle, the irradiated area is defined by the linear track of the HZE particle and is in the submicrometer range (6-9). Low-LET radiation microbeams, such as the ones using ultrasoft $X$ rays, typically employ a collimator mask consisting of a metal grid placed on an X-ray transparent polymer film (10-13). Metal grids can be constructed by lithography with aperture dimensions of $1 \mu \mathrm{m}$ or less, but, due to fabrication constraints, the thickness of these grids is also limited to a few micrometers. In turn, this necessitates the use of ultrasoft $X$ rays, which can be blocked by a few micrometers of metal. Further, because ultrasoft $\mathrm{X}$ rays can be scattered easily and have limited penetrance in air and liquids, the cells have to be positioned within a few micrometers of the grid for the collimation to be effective (10-13).

The development of all the microbeams discussed above requires significant expertise and resources. Further, in many cases, a particle accelerator is required for their function. As a result, currently, there are only about 30 microbeams in operation or under development worldwide (5), and alternate methods that rely on UV lasers are becoming popular for local induction of DNA damage $(3,4)$.

We describe here a collimator that can be used with radiation produced by conventional high-voltage $\mathrm{X}$-ray tubes and that is suitable for irradiating areas whose dimensions 
are in the micrometer range. This novel microcollimator provides good masking contrast for X-ray energies of up to $30 \mathrm{keV}$ and has very small collimation angles, enabling it to be positioned even a few millimeters away from the irradiated target without pattern blurring.

\section{MATERIALS AND METHODS}

\section{Silicon Chip Fabrication}

The silicon chips were fabricated at the Center of MicroNanoTechnology at EPFL. Standard 380- $\mu$ m-thick double-side polished silicon wafers were used as the X-ray attenuating material in the microcollimators. The surfaces of the wafers were coated with sacrificial oxide layers with a thickness of $0.5,1,2$ or $10 \mu \mathrm{m}$ that would later be etched to generate grooves of the same depths in the microcollimator. A photoresist was then patterned in stripes on the top side of the silicon wafers using conventional photolithography. The photoresist served as an etch-mask so that the $\mathrm{SiO}_{2}$ could be etched down to the silicon using either buffered $\mathrm{HF}$ etching in the case of the 0.5-, 1- and 2- $\mu \mathrm{m}$ oxides or reactive ion etching in the case of the $10-\mu \mathrm{m}$ oxide. Based on the photoresist patterns, the grooves produced by the etching had widths of $0.1,0.25,0.5,0.8$ or $3.5 \mathrm{~mm}$. After etching, the wafers were diced into chips that were eventually stacked in the microcollimator. Two sizes of chips were diced: 1 $\mathrm{cm} \times 1 \mathrm{~cm}$ and $2 \mathrm{~cm} \times 1 \mathrm{~cm}$. After dicing, the photoresist was stripped with acetone and the wafers were rinsed in deionized water before being cleaned by oxygen plasma.

\section{Fabrication of the Microcollimator Holder}

The microcollimator holder was fabricated at the Department of Molecular Biology of the University of Geneva. A stainless steel block in the shape of a cube with a rectangular-shaped hole in the center was constructed to hold the stacked silicon chips, which were pressed against one wall of the hole by a metal plate held under tension by a screw. A cell holder made of stainless steel and teflon was constructed to hold the 18-mm-diameter glass cover slips on which cells could be cultured firmly. The cell holder was attached to the silicon chip holder, such that the cells would be positioned about $0.5 \mathrm{~mm}$ away from the outlet surface of the silicon chips. As a unit, the cell holder and the silicon chip holder were attached by a 1:120,000 ratio gearbox to a high-precision stepper motor, allowing smooth rotation of the microcollimator over a wide range of speeds. The rotation of the microcollimator was computer-controlled using digital position sensors incorporated in the microcollimator.

\section{Calculation of X-Ray Attenuation}

The level of attenuation of $X$ rays of various energies as a function of the length of the silicon chips was calculated using the Beer-Lambert law and data obtained from the NIST database X-ray attenuation tables for silicon and $\mathrm{SiO}_{2}$ (14). Monte Carlo simulations for estimating radiation exposure rates were performed using a computer program written specifically for this purpose. The geometry of the microcollimator, the target and the anode focal spot were reproduced by the program and the paths and intensities of 10,000 photons emanating randomly from the anode focal spot were calculated.

\section{Irradiation of Cells}

The microcollimator was attached to the collimator holder of an XRAD 320 Irradiator (Precision X-Ray, Inc., North Branford, CT), which uses an MXR-321 X-ray tube (Comet, Flamatt, Switzerland). Glass cover slips on which U2OS human osteosarcoma cells were cultured were placed on the cell holder of the microcollimator and were covered with tissue culture medium. The microcollimator was then programmed to perform a slow rotation over a $2-4^{\circ}$ angle range, such that at the rotation midpoint the cover slip would be facing the focal spot of the anode. The rotation was programmed to last 6-15 min, and during this time the X-ray tube was turned on. The settings for the X-ray tube were 20 or $30 \mathrm{keV}$ at $25 \mathrm{~mA}$. After irradiation the cells were returned to the tissue culture incubator for $30 \mathrm{~min}$ to allow robust 53BP1 focus formation and were then fixed and processed for immunofluorescence, as described previously (15).

\section{RESULTS}

\section{Device Description and Manufacturing Process}

The microcollimator consists of a stack of X-ray attenuating silicon chips with grooves lithographically defined and etched along their upper surfaces (Fig. 1A). When the chips are stacked, these grooves create collimating slits through which $\mathrm{X}$ rays can pass (Fig. 1B).

The silicon chips were manufactured starting with standard 380- $\mu$ m-thick double-side polished silicon wafers (typically used in microfabrication). The surfaces of the wafers presented sacrificial silicon dioxide $\left(\mathrm{SiO}_{2}\right)$ layers that would later serve as spacers to establish the grooves in the microcollimators. $\mathrm{SiO}_{2}$ layers of $0.5,1,2$ or $10 \mu \mathrm{m}$ were used to produce microcollimators with slit widths of the same dimensions (Fig. 1B). A photoresist was patterned in stripes on the top side of the silicon wafers using conventional photolithography. The photoresist served as an etch-mask so that the $\mathrm{SiO}_{2}$ could be etched down to the silicon using either buffered HF etching in the case of the $0.5-, 1-$ and $2-\mu \mathrm{m}$ oxides or reactive ion etching in the case of the $10-\mu \mathrm{m}$ oxide. After etching, which produced grooves of depths of $0.5,1,2$ or $10 \mu \mathrm{m}$ and widths of $0.1,0.25$, $0.5,0.8$ or $3.5 \mathrm{~mm}$ (Fig. 1A), the wafers were diced into chips with dimensions of $1 \mathrm{~cm} \times 1 \mathrm{~cm}$ or $2 \mathrm{~cm} \times 1 \mathrm{~cm}$ (length $\times$ width).

The quality of the silicon chip manufacturing process was evaluated by several methods. Scanning electron microscopy (SEM) images showed well-defined grooves with smooth edges in spite of the dicing saw (Fig. 1C and D). The depth of the grooves on the surfaces of the chips was measured using a surface stylus profilometer (Tencor) and the thickness of the non-etched $\mathrm{SiO}_{2}$ spacer using a surface reflectometer (Nanometrics). A comparison of the two sets of measurements showed a very good correlation, indicating that the original thickness of the $\mathrm{SiO}_{2}$ film effectively determined the depth of the microcollimator grooves (Table 1).

After manufacturing, enough silicon chips were stacked against each other to create a block that was about $1.5 \mathrm{~cm}$ wide, so that cells growing on 18-mm-diameter cover slips could be irradiated. Tight conformal stacking of the chips was ensured by a microcollimator holder (Fig. 2A). The latter consists of a stainless steel block with a rectangularshaped hole at its center, where the stacked silicon chips were positioned. The chips were pressed against one wall of the hole by a stainless steel plate held in place by a screw attached to the microcollimator holder (Fig. 2A). Once the silicon chips were stacked, the grooves on their surfaces led to the formation of slits, which could be vi- 

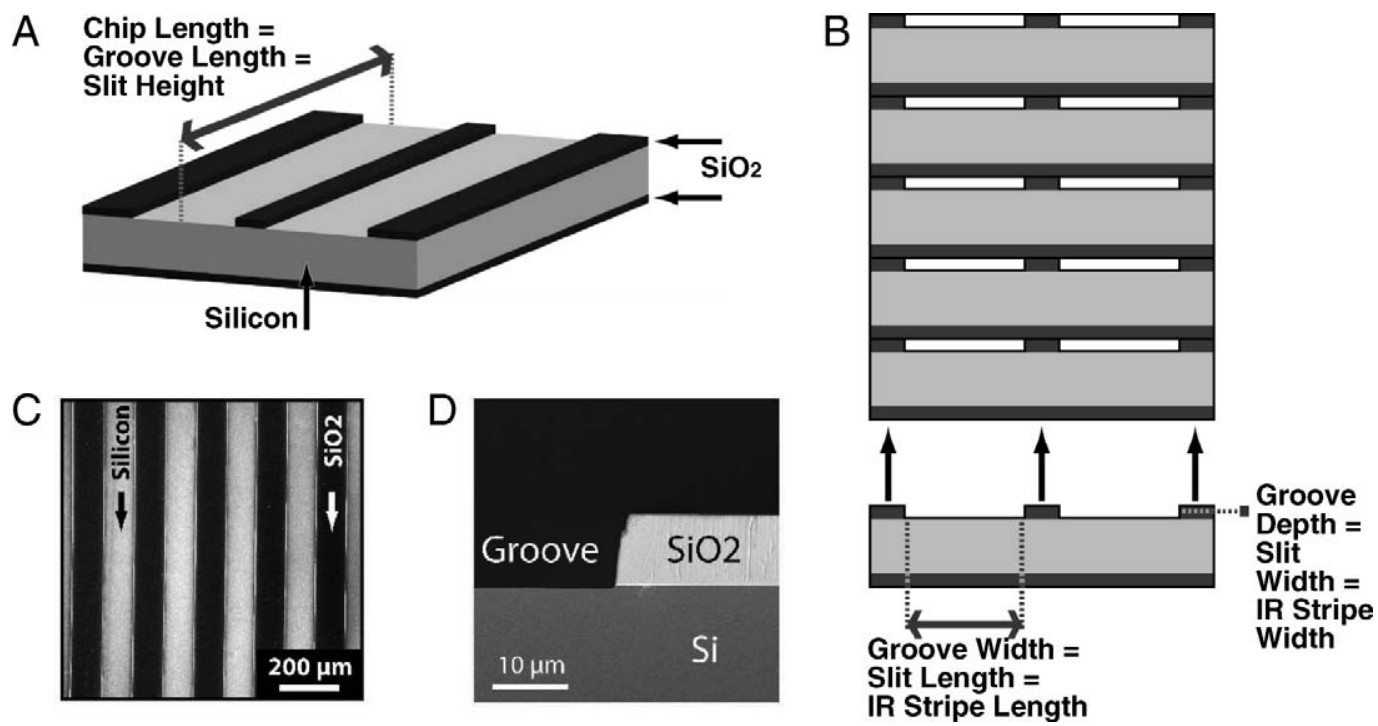

FIG. 1. Design and quality control assessment of silicon chips used in the microcollimator. Panel A: Diagram of a single silicon chip showing the surface layers of $\mathrm{SiO}_{2}$. On the top surface, parts of the $\mathrm{SiO}_{2}$ layer have been etched to form grooves. Panel B: Stacking of silicon chips creates slits through which X rays can pass to generate a pattern of irradiation (IR) stripes on the target. Panel C: Scanning electron micrograph image of the top surface of a silicon chip showing a series of $100-\mu \mathrm{m}$-wide grooves. Panel D: Scanning electron micrograph image of the end view of the wall of a $10-\mu \mathrm{m}$-deep groove. The remaining $\mathrm{SiO}_{2}$ thin film serving as a spacer is visible on the right. On the left, the base of the groove is smooth where the $\mathrm{SiO}_{2}$ was etched away from the silicon.

sualized under a microscope by allowing light to pass through the microcollimator (Fig. 2B).

\section{Geometry Considerations and Radiation Dose Estimates}

The stripe pattern produced by the microcollimator is the result of the difference in X-ray attenuation between the silicon chips and the air in the slits. For the case of an incident monochromatic X-ray beam parallel to the microcollimator channels, the resulting contrast is given by the Beer-Lambert law:

$$
C=I_{T} /\left(I_{O} e^{-T / L}\right)
$$

where $I_{O}$ is the intensity at the inlet surface of the microcollimator, $I_{T}$ is the intensity at the outlet surface, $T$ is the thickness of the microcollimator, and $L$ is the material attenuation length for the X-ray energy in question. Plotting the contrast between the grooves and attenuating portions of the microcollimator as a function of the length of the attenuating material and of the energy of the incipient $\mathrm{X}$ rays showed that 1 - and 2-cm-long chips would block more than $99 \%$ of $\mathrm{X}$ rays with energies of $23 \mathrm{keV}$ and $30 \mathrm{keV}$, respectively (Fig. 3).

Based on these calculations, the silicon chips were manufactured to be 1 or $2 \mathrm{~cm}$ long. The length of the chips in turn dictates the geometry of the X-ray collimating slits. The height of the slits is equal to the length of the silicon chips ( 1 or $2 \mathrm{~cm}$ ), their width is equal to the depth of the grooves on the surface of the chips $(0.5-10 \mu \mathrm{m})$, and their length is equal to the width of the grooves $(0.1-3.5 \mathrm{~mm})$ (Fig. 1A and B). Taking into account the dimensions of width and height, these slits collimate $\mathrm{X}$ rays to within angles in the range of $0.0014-0.0573^{\circ}$ (Fig. 4A). An advantage of the small collimation angle (ANco) is that the $\mathrm{X}$ rays coming out of the collimator are essentially parallel, which means that the biological target can be placed even 1-2 mm away from the collimator. However, there are two disadvantages to the small collimation angle. The first disadvantage is that it limits the part of the anode focal spot that is "visible" from the target and hence results in decreased exposures. The decrease in the exposure can be estimated by considering that in the absence of the microcollimator, the target is exposed to $\mathrm{X}$ rays emanating from the entire anode focal spot. In this case, the unobstructed angle (ANun) represents the angle through which the focal spot is visible from the target and through which the target

TABLE 1

Quality Assessment of the Depth of the Microfabricated Grooves on the Surfaces of the Silicon Chips

\begin{tabular}{ccc}
\hline $\begin{array}{c}\text { Desired } \\
\text { groove depth } \\
(\mu \mathrm{m})\end{array}$ & $\begin{array}{c}\text { Measured } \\
\mathrm{SiO}_{2} \text { film thickness } \\
(\mathrm{nm})\end{array}$ & $\begin{array}{c}\text { Measured } \\
\text { groove depth } \\
(\mathrm{nm})\end{array}$ \\
\hline 10 & $10,150 \pm 12$ & $10,550 \pm 300$ \\
1 & $1,006 \pm 7$ & $1,083 \pm 79$ \\
0.5 & $507 \pm 6$ & $550 \pm 66$ \\
\hline
\end{tabular}

Notes. $\mathrm{SiO}_{2}$ film thickness was measured with a surface reflectometer, while groove depth was measured with a surface profilometer. The $0.5-$ and $1-\mu \mathrm{m}$ groove depths correspond well to their respective film thicknesses. The $10-\mu \mathrm{m}$ groove depth was larger than the film thickness, indicating that some of the silicon below the $\mathrm{SiO}_{2}$ film was also removed during the etching process. Means \pm 2 SD of 10 independent measurements are given. 
A

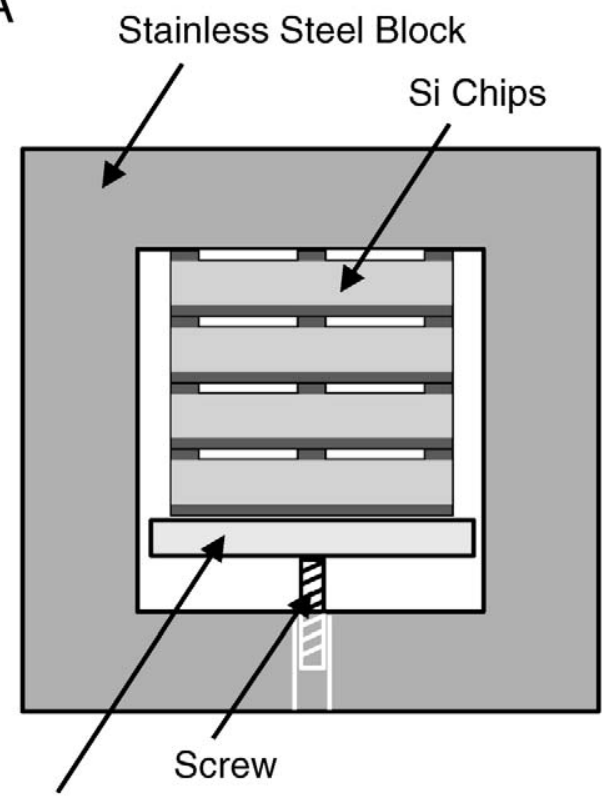

B
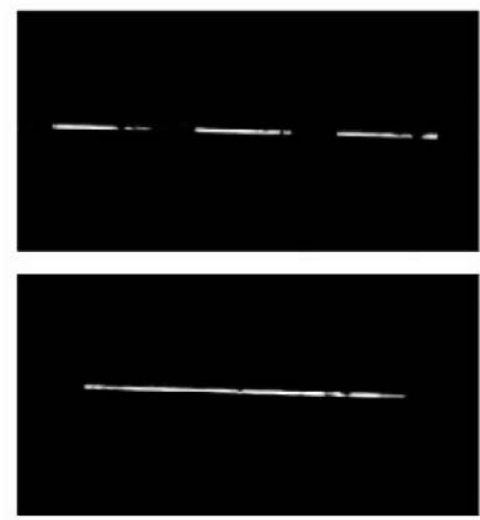

\section{Stainless Steel Plate}

FIG. 2. Packing of silicon chips in the holder produces slits dimensions that match the dimensions of the etched grooves. Panel A: Diagram of stacked silicon chips in the holder. The chips are pressed against one wall of the rectangle-shaped hole in the holder by a stainless steel plate. Tension is generated by a screw. Panel B: Microscope images of light passing through the slits formed by stacked silicon chips. The slits are $10 \mu \mathrm{m}$ wide and either 250 $\mu \mathrm{m}$ or $800 \mu \mathrm{m}$ long (upper and lower images, respectively).

is exposed to $\mathrm{X}$ rays. Assuming that the attenuation length of the silicon wafers is zero (i.e., no $\mathrm{X}$ rays can penetrate even the slightest thickness of silicon) and that the intensity of generated $\mathrm{X}$ rays is uniform throughout the anode focal spot, then the exposure dose of the target at the outlet of a collimator slit (DOco) would be related to the dose received by an unobstructed target (DOun) by the formula

$$
\text { DOco }=\text { DOun(ANco/ANun). }
$$

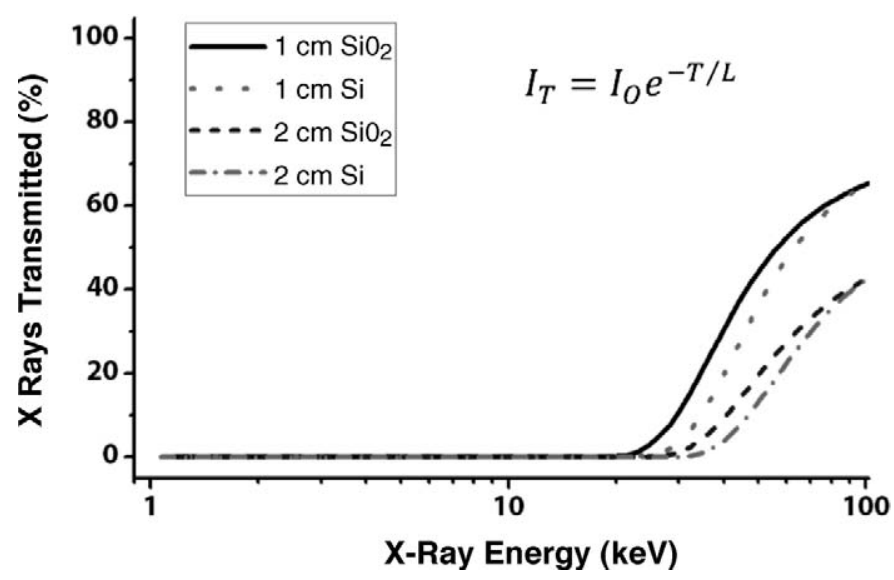

FIG. 3. Calculated intensities of $X$ rays transmitted through 1 - and 2 -cm-long microcollimators as a function of incident X-ray energy. The failure point was defined as the X-ray energy (in $\mathrm{keV}$ ) for which the intensity of transmitted $\mathrm{X}$ rays exceeded $1 \%$ of the incipient intensity. The failure points were: $1 \mathrm{~cm} \mathrm{SiO} 2,22.98 \mathrm{keV} ; 1 \mathrm{~cm}$ silicon, $28.08 \mathrm{keV}$; $2 \mathrm{~cm} \mathrm{SiO}_{2}, 30.01 \mathrm{keV} ; 2 \mathrm{~cm}$ silicon, $36.67 \mathrm{keV}$.
The term DOun can be easily measured experimentally. For the X-ray tube we used in this study (Comet, MXR-321) at settings of 20 or $30 \mathrm{keV}$ and $25 \mathrm{~mA}$ and a target to anode focal spot distance of $25 \mathrm{~cm}$, the DOun is 7 and 15 Gy/min, respectively. The angle ANun can also be estimated. The diameter of the focal spot of this X-ray tube is $8 \mathrm{~mm}$ and its target angle is $30^{\circ}$. Thus, when viewed from the target, the size of the focal spot is $4 \mathrm{~mm}$ and at a 25$\mathrm{cm}$ target to anode focal spot distance, ANun equals $0.91^{\circ}$. Since ANco is much smaller than ANun, the exposure of a target at the outlet of a collimator slit is much smaller than the exposure of an unobstructed target.

Fortunately, silicon is not impermeable to $20-30 \mathrm{keV} \mathrm{X}$ rays. Thus some $\mathrm{X}$ rays that penetrate the silicon and $\mathrm{SiO}_{2}$ walls of the slit can reach the target, resulting in increased exposures (Fig. 4C). To determine a more realistic estimate of the exposure of a target at the outlet of a collimator slit, we performed Monte Carlo simulations taking into account the geometry of the microcollimator and the attenuation lengths of silicon and $\mathrm{SiO}_{2}$ for 20 and $30 \mathrm{keV}$ X rays. The simulations were performed for slit widths of $0.5,1,2,5$ or $10 \mu \mathrm{m}$ and slit heights of 1 or $2 \mathrm{~cm}$. The results predicted increased exposure compared to the one calculated for zero attenuation length (Table 2). As expected, the increase was greater for narrow slits $(0.5-2 \mu \mathrm{m})$ than for wide slits $(5-10 \mu \mathrm{m})$ and for $30 \mathrm{keV}$ than for $20 \mathrm{keV}$. We also calculated the exposure of a target placed below the silicon wafer, away from a slit. At $30 \mathrm{keV}$, the 1 -cm-long wafers were predicted to be penetrated by the $\mathrm{X}$ rays (Table 

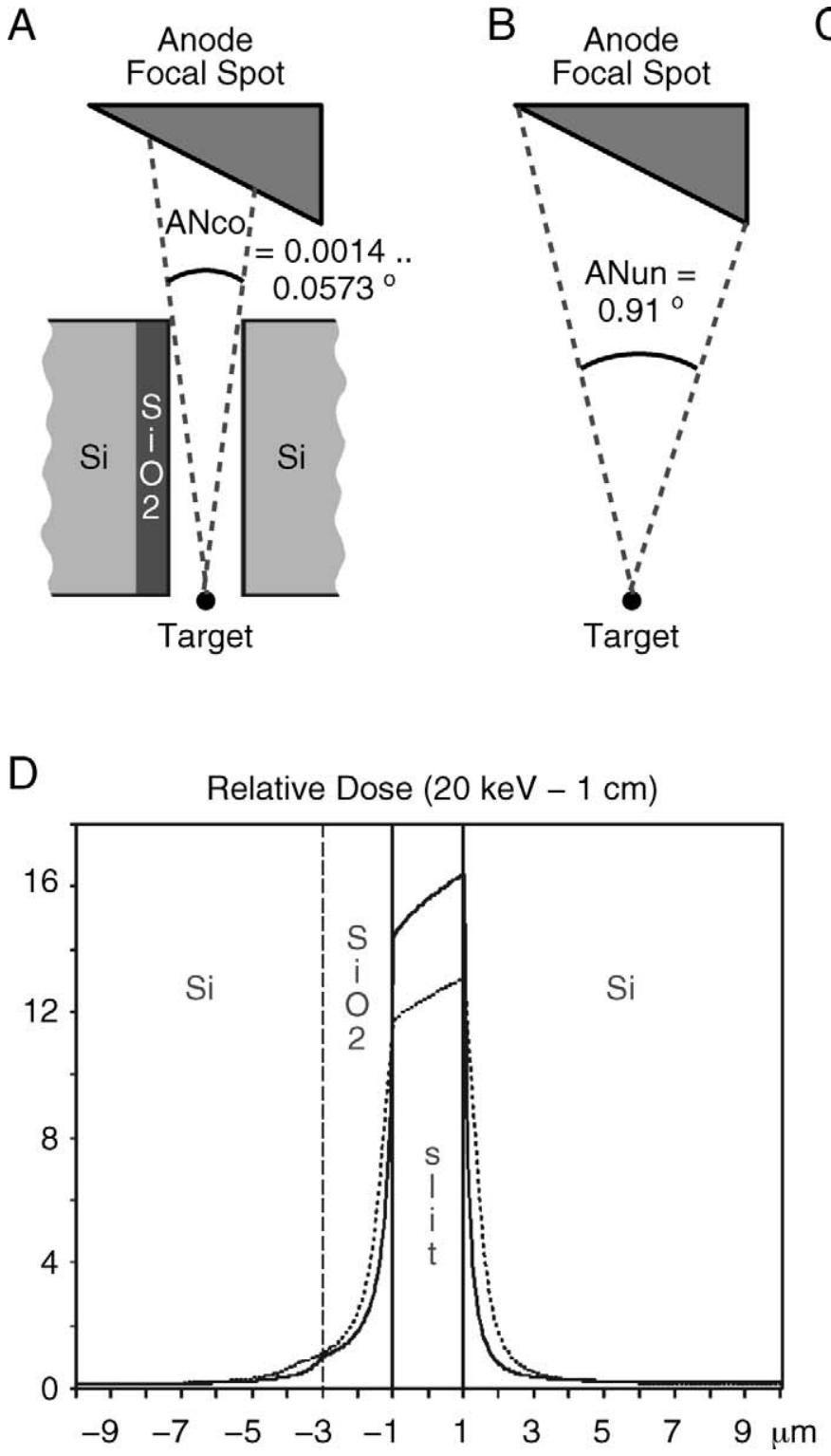

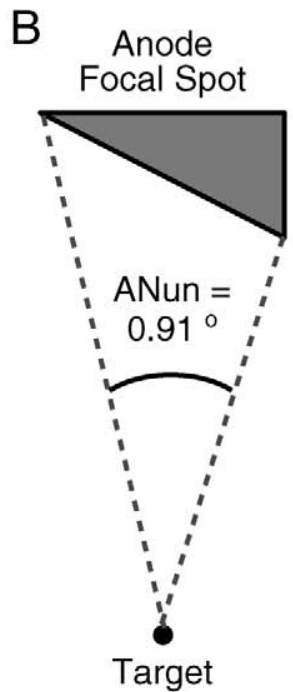

Target
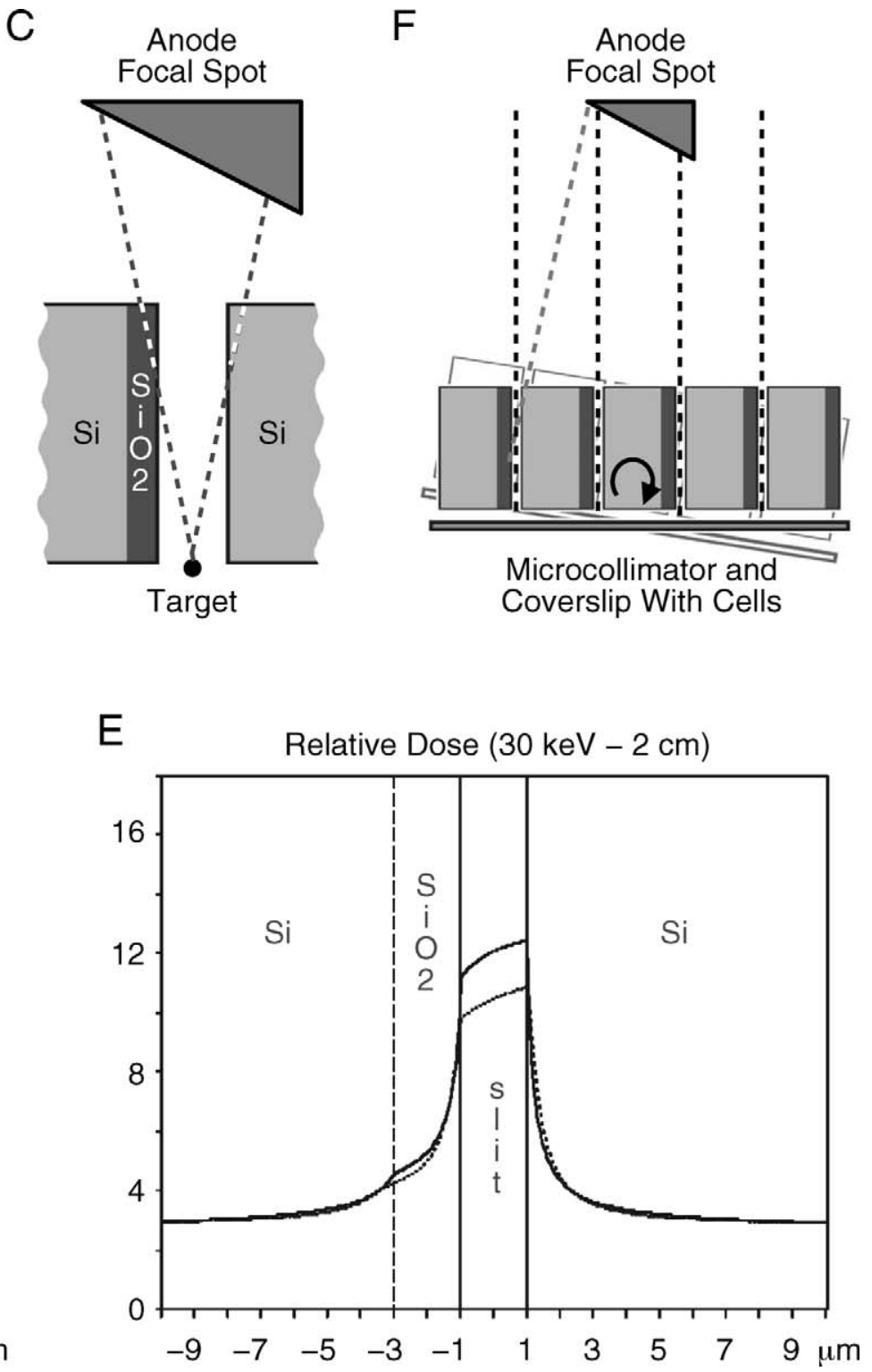

FIG. 4. Geometry considerations of the microcollimator design and estimation of radiation exposure. Panel A: The collimation angle (ANco) is determined by the width $(0.5-10 \mu \mathrm{m})$ and height $\left(1-2 \mathrm{~cm}\right.$ ) of the collimating slits and ranges between $0.0014-0.0573^{\circ}$ (in the configurations we have constructed to date). Because the collimation angle is very small, a target at the outlet of a slit is exposed to X rays emitted from only part of the anode focal spot. Panel B: A target with no obstruction between it; the anode focal spot is exposed to X rays emanating from the entire focal spot. The unobstructed angle (ANun) represents the angle through which the focal spot is visible from the target and through which the target is exposed to $\mathrm{X}$ rays. In the configuration we used, ANun is $0.91^{\circ}$. Panel C: Since the silicon wafers are partially transparent to $\mathrm{X}$ rays, the exposure of the target is greater than the one calculated solely on the basis of the collimating angle (ANco). Panel D: Monte Carlo simulations of exposure for targets 0 mm (solid line) or $2 \mathrm{~mm}$ (dotted line) below the surface of the microcollimator. Results are presented as parts per thousand of the unobstructed exposure (i.e., the unobstructed exposure is set to 1,000). The simulations were performed for $20 \mathrm{keV} \mathrm{X}$ rays, for a $2-\mu \mathrm{m}$-wide and 1-cm-high slit and for a segment of the microcollimator extending $10 \mu \mathrm{m}$ either side from the center of the slit. Panel E: Monte Carlo simulations as for panel D, except that the X-ray energy was set at $30 \mathrm{keV}$ and the slit height at $2 \mathrm{~cm}$. Panel F: The small collimation angle and the small size of the anode focal spot relative to the width of the microcollimator make it impossible for all slits to point to the X-ray source at the same time. Rotation of the microcollimator along the indicated axis during irradiation ensures that all slits point to the anode focal spot.

2), as also predicted by Beer-Lambert's law (Fig. 3). Finally, we also calculated the exposure along the entire width of a slit extending to the adjacent outlet surface microcollimator to get dose contrast estimates. These calculations were performed for targets at a distance of 0 or 2 $\mathrm{mm}$ from the outlet surface of the microcollimator. Very high-contrast and sharp radiation stripe boundaries were predicted with $20 \mathrm{keV} X$ rays, even for targets that were 2 $\mathrm{mm}$ away from the outlet surface of the microcollimator (Fig. 4D). The performance of the microcollimator was predicted to decrease with $30 \mathrm{keV} \mathrm{X}$ rays but was still satisfactory (Fig. 4E). It should be noted that our calculations 
TABLE 2

Calculation of Radiation Exposure at the Outlet of the Microcollimator Slits

\begin{tabular}{|c|c|c|c|c|}
\hline & & \multicolumn{3}{|c|}{$\begin{array}{l}\text { Exposure relative to unobstructed exposure } \\
\qquad(=1,000)\end{array}$} \\
\hline \multicolumn{2}{|c|}{ Slit dimensions } & & Attenuation & Attenuation \\
\hline $\begin{array}{l}\text { Height } \\
(\mathrm{cm})\end{array}$ & $\begin{array}{l}\text { Width } \\
(\mu \mathrm{m})\end{array}$ & $\begin{array}{l}\text { Attenuation } \\
\text { length }=0\end{array}$ & $\begin{array}{l}20 \mathrm{keV} \\
\text { silicon/SiO }\end{array}$ & $\begin{array}{c}30 \mathrm{keV} \\
\text { silicon } / \mathrm{SiO}_{2}\end{array}$ \\
\hline 1 & 0.5 & 3.20 & 3.96 & 58.34 \\
\hline 1 & 1 & 6.30 & 7.84 & 66.37 \\
\hline 1 & 2 & 12.50 & 15.63 & 81.48 \\
\hline 1 & 5 & 31.30 & 38.94 & 123.22 \\
\hline 1 & 10 & 62.50 & 77.76 & 186.76 \\
\hline 1 & off-slit & 0.00 & 0.08 & 49.47 \\
\hline 2 & 0.5 & 1.60 & 1.72 & 4.91 \\
\hline 2 & 1 & 3.20 & 3.40 & 7.31 \\
\hline 2 & 2 & 6.30 & 6.82 & 12.08 \\
\hline 2 & 5 & 15.70 & 17.07 & 26.24 \\
\hline 2 & 10 & 31.30 & 34.13 & 49.53 \\
\hline 2 & off-slit & 0.00 & 0.00 & 2.64 \\
\hline
\end{tabular}

Notes. Computer simulations were performed assuming an 8-mm-diameter anode focal spot with a $30^{\circ}$ target angle; the target was placed 0 $\mathrm{mm}$ below the outlet of the slit and a distance of $25 \mathrm{~cm}$ between the target and the anode focal spot. The simulations were performed with X-ray attenuation lengths for silicon and $\mathrm{SiO}_{2}$ obtained from the NIST database (14) or with an attenuation length of zero to determine whether $\mathrm{X}$ rays did not penetrate the silicon. The exposure of the target is represented as parts per thousand of the unobstructed exposure (i.e., the unobstructed exposure is set to 1,000). Exposures were calculated for targets placed at the center below slits of the indicated dimensions or below the silicon away from the slit (off-slit) to show to what degree the $\mathrm{X}$ rays penetrate the silicon wafers.

apply to monochromatic $\mathrm{X}$ rays, which of course is not true for $\mathrm{X}$ rays emitted by high-voltage $\mathrm{X}$-ray tubes. This means that the actual exposure contrast at the $30 \mathrm{keV}$ setting will be better than our simulations indicate (see below).

The second disadvantage of the very small collimation angle is that it becomes difficult to align the microcollimator to the focal spot of the anode of the X-ray tube. In fact, given that the size of the focal spot of a typical X-ray tube, when viewed from the target, is about $4 \mathrm{~mm}$, it is impossible for $X$ rays to pass through all the slits of a 1.5cm-wide microcollimator at the same time (Fig. 4F). To resolve this problem, the microcollimator holder was attached to a motor that allowed it to be rotated with an axis of rotation perpendicular to the surface of the silicon chips and to the X-ray beam. By rotating the microcollimator holder over a $3.2^{\circ}$ range, we ensured (for a $1.5-\mathrm{cm}$-wide microcollimator positioned $25 \mathrm{~cm}$ from the focal spot) that at some point during this rotation every slit would become aligned to the anode focal spot (Fig. 4F). A cell platform, able to hold an 18-mm-diameter cover slip with cells, was attached to the microcollimator holder, such that the cells would be positioned about $0.5 \mathrm{~mm}$ away from the outer surface of the microcollimator and would rotate as a single body with the microcollimator (Fig. 4F).

\section{Validation of the Microcollimator}

We set out to validate the microcollimator by irradiating human U2OS cells and monitoring by immunofluorescence the intracellular localization of 53BP1, a protein that is rapidly recruited to sites of DNA DSBs $(15-18)$. We used a conventional high-voltage X-ray tube (Comet, Flamatt, Switzerland) to generate $\mathrm{X}$ rays with a current setting of $25 \mathrm{~mA}$ and voltage settings of 10, 15, 20,30, 40 and 50 $\mathrm{keV}$. At 10 and $15 \mathrm{keV}$, the $\mathrm{X}$ rays were unable to penetrate the exit window of the $\mathrm{X}$-ray tube. At $20 \mathrm{keV}$, the $\mathrm{X}$ rays were efficiently collimated by the microcollimator with silicon chip lengths of either 1 or $2 \mathrm{~cm}$. At $30 \mathrm{keV}$, the $\mathrm{X}$ rays were efficiently collimated by the microcollimator with 2-cm-long silicon chips but penetrated the microcollimator with 1-cm-long silicon chips, as predicted by our calculations (Fig. 3 and Table 2), while at 40 and $50 \mathrm{keV}$, the $\mathrm{X}$ rays penetrated both the 1- and 2-cm-long chips. Examples of the 53BP1 immunofluorescence patterns achieved by a microcollimator with $2-\mathrm{cm}$-high and $2-\mu \mathrm{m}$-wide slits are shown in Fig. 5. These patterns validate the ability of the microcollimator to limit the irradiated area to stripes, whose width is in the micrometer range.

Based on the simulations described above, we calculated the exposure at the outlet of the collimator slits for the experiment shown in Fig. 5. We experimentally measured the dose rate in the absence of the collimator (DOun) $25 \mathrm{~cm}$ away from the anode focal spot and found it to be $15 \mathrm{~Gy} / \mathrm{min}$ (for settings of $30 \mathrm{keV}$ and $25 \mathrm{~mA}$ ). Based on the simulations of Table 2 (for 2-cm-high and 2- $\mu$ m-wide slits), the dose rate at the outlet of the slits should be $0.18 \mathrm{~Gy} / \mathrm{min}$. The cells were irradiated for $6 \mathrm{~min}$, which puts the exposure at $1.1 \mathrm{~Gy}$. The calculated exposure is consistent with the number of 53BP1 foci observed in these cells (Fig. 5), given that 1 Gy of radiation results in about 20-25 53BP1 foci per cell when the entire nucleus is irradiated (15).

\section{DISCUSSION}

We describe here the development of a microcollimator that allows subnuclear compartments of cells to be exposed to low-LET radiation. The main advantage of this microcollimator is its simplicity of use and its compatibility with conventional high-voltage X-ray tubes.

Previously described low-LET radiation microbeams either were designed to irradiate single cells to study bystander effects or, when it was desired to irradiate part of a cell, employed thin X-ray masks to collimate the beam. These masks contain X-ray absorbing metallic regions, for example, made of gold, layered on an X-ray transparent polymer (10-13). Due to fabrication constraints on the aspect ratios of the metallic X-ray absorbing components, the thickness of the metal in these masks is about $1 \mu \mathrm{m}$. In turn, this affects the entire design of the microbeam. When $\mathrm{X}$ rays are used as the source of low-LET radiation, their energy must be low enough, for example $1.34 \mathrm{keV}$ in one 

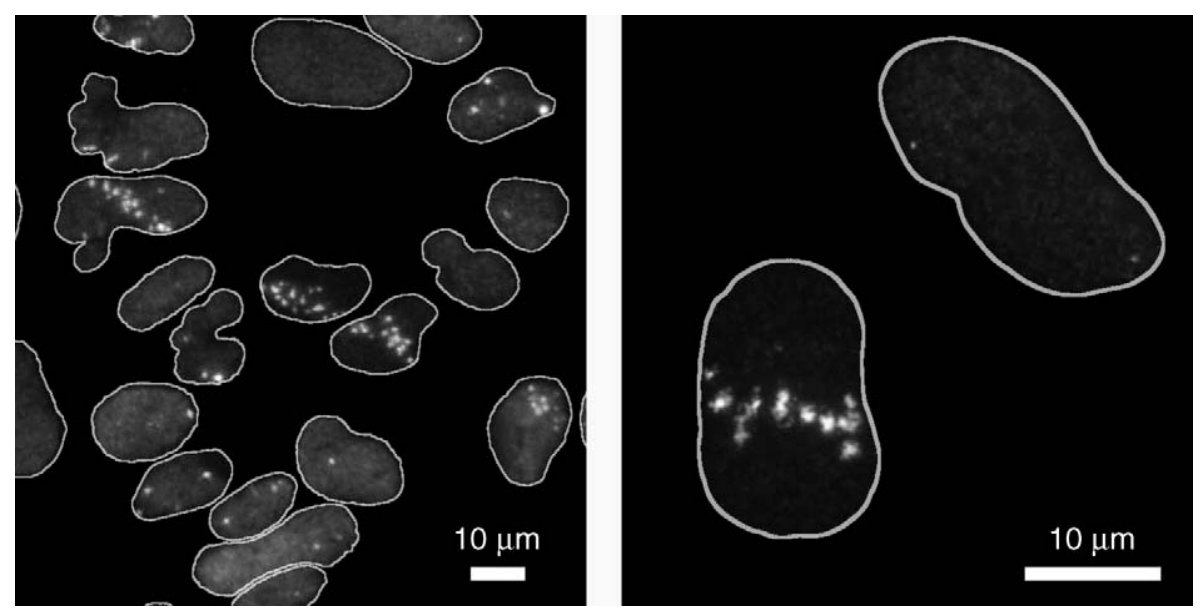

FIG. 5. 53BP1 is recruited to stripes after collimation of the X-ray beam with a microcollimator containing 2 $\mathrm{cm}$-long silicon chips with $2-\mu \mathrm{m}$-wide slits. U2OS cells were exposed to $\mathrm{X}$ rays using a conventional high-voltage $\mathrm{X}$-ray tube and settings of $30 \mathrm{keV}$ and $25 \mathrm{~mA}$. The cells were irradiated for $6 \mathrm{~min}$, during which time the microcollimator was rotated over a $2^{\circ}$ angle, and were processed for immunofluorescence $30 \mathrm{~min}$ after irradiation. The periphery of the nuclei is indicated by gray lines. The two images are at $40 \times$ (left) and $100 \times$ (right) magnification.

case $(10,11)$, so that they can be blocked by the thin metallic component. Such ultrasoft X rays cannot penetrate the exit window of conventional high-voltage $\mathrm{X}$-ray tubes and therefore require specialized $\mathrm{X}$-ray sources, such as synchrotron particle accelerators. Further, these ultrasoft X rays have very low penetrance in air and biological materials, which means that the cells to be irradiated have to be placed within a few micrometers of the X-ray mask.

One way to overcome the requirement for ultrasoft $X$ rays is to increase the thickness of the X-ray absorbing metallic component. For gold, a thickness of about $80 \mu \mathrm{m}$ would be necessary to successfully mask $20 \mathrm{keV} X$ rays. It is now becoming technically feasible to fabricate $1-\mu \mathrm{m}$ wide polymer structures with heights of up to $80 \mu \mathrm{m}$ (19), and such structures could be used, in principle, to generate gold masks thick enough for use with conventional X-ray tubes. However, such a collimator would still have a relatively small aspect ratio (80:1), which for non-parallel $\mathrm{X}$-ray beams such as the ones produced by high-voltage $\mathrm{X}$-ray tubes would mean that the cells would still have to be positioned just a few micrometers away from the mask to avoid pattern blurring.

The X-ray microcollimator described here allows the use of conventional high-voltage X-ray tubes and is compatible with placing the target cells even a few millimeters away from the collimator. The ability of our microcollimator to absorb $\mathrm{X}$ rays is not limited by a fabrication process aspect ratio, unlike the case of X-ray masks, because the width of the slits is a function of the thickness of the thin film coating the silicon chips, while the thickness of the absorber is defined by the length of the chips when they are diced, which is a separate fabrication step. Thus the fabrication method we present here enables us to increase the thickness of the absorber just by dicing longer chips. A second advantage of our method is that the chip length $(1$ or $2 \mathrm{~cm})$ is much greater than the slit width $(0.5-10 \mu \mathrm{m})$, resulting in very small collimation angles. Thus, even though the beam emitted by X-ray tubes is not parallel, the beam exiting the collimator is almost parallel. Combined with the fact that the distance between slits is quite large $(380 \mu \mathrm{m}$, corresponding to the thickness of the silicon chips), this means that the cells can be placed even a few millimeters away from the collimator. In turn, this allows us to grow the cells on standard glass cover slips, rather than on special supports, such as micrometer-thick mylar membranes.

Another advantage of our system is that it can be adapted to harder $\mathrm{X}$ rays just by changing the material in the chips. For example, if GaAs is used in place of silicon, then a 2-cm microcollimator could effectively mask electrons with energies as high as $180 \mathrm{keV}$. One concern, however, with using such high-energy $X$ rays is that while $X$ rays in the 1-30 keV range interact with matter mostly through the photoelectric effect, the interaction of matter with harder X rays becomes dominated by Compton scattering (13). Secondary electrons produced from photoelectric absorption have limited penetrating power in biological tissues, which effectively ensures that only the part of the cell exposed to photons of $X$ rays suffers radiation damage. In contrast, the energetic electrons produced by Compton scattering have higher energies and will result in less localized damage, thereby compromising our ability to specifically target subnuclear compartments.

The final advantage of the microcollimator described here is its ease of use. The microcollimator can simply be attached to a conventional high-voltage $\mathrm{X}$-ray tube, a cover slip with cells can be placed on the cell holder, and the motor can then be programmed to rotate the microcollimator and cells, while the X-ray beam is turned on. Attaching the microcollimator to an inverted microscope (which would have to be placed in the irradiation cabinet 
and operated remotely) to allow the cells to be visualized during irradiation is also possible, although we have not yet implemented this option. The ease of use of this microcollimator may allow greater access of subnuclear irradiation to the scientific community. Previously developed microbeams require significant resources and expertise, which led many investigators to induce localized DNA damage using UV lasers on cells that had been presensitized by incubation with Hoechst dyes or by incorporating bromodeoxyuridine (BrdU) in their DNA $(3,4)$. While the UV laser approach has many advantages, it also has disadvantages. The spectrum of DNA damage lesions induced by the UV laser includes pyrimidine dimers and most likely protein-DNA crosslinks, in addition to single-stranded and double-stranded DNA breaks (20-22). More importantly, the Hoechst dyes and the BrdU incorporation used to presensitize the cells may result in aberrant responses to DNA damage and produce radiosensitization (23-25). In fact, Hoechst dyes, even in the absence of exposure to UV light, can change the conformation of DNA, leading to chromosome decondensation and transcription inhibition $(26,27)$.

In conclusion, we have successfully demonstrated a simple microcollimator for applications in stripe irradiation. The simple design of the microcollimator ensures that it can be easily adapted to existing X-ray tubes, facilitating the study of cellular responses to low-LET radiation.

\section{ACKNOWLEDGMENTS}

The authors thank the Center of MicroNanoTechnology at EPFL and Yves-Alain Poget, Marc Schneider and Georges Severi at the University of Geneva for technical support. This work was supported by grants from the Swiss National Science Foundation to KP and TDH.

Received: May 30, 2008; accepted: October 10, 2008

\section{REFERENCES}

1. J. B. Little, Cellular effects of ionizing radiation. N. Engl. J. Med. 278, 369-376 (1968).

2. D. T. Goodhead, Initial events in the cellular effects of ionizing radiations: clustered damage in DNA. Int. J. Radiat. Biol. 65, 7-17 (1994).

3. C. Lukas, J. Bartek and J. Lukas, Imaging of protein movement induced by chromosomal breakage: tiny 'local' lesions pose great 'global' challenges. Chromosoma 114, 146-154 (2005).

4. J. Essers, W. Vermeulen and A. B. Houtsmuller, DNA damage repair: anytime, anywhere? Curr. Opin. Cell Biol. 18, 240-246 (2006).

5. D. J. Brenner and E. J. Hall, Microbeams: a potent mix of physics and biology. Summary of the 5th International Workshop on Microbeam Probes of Cellular Radiation Response. Radiat. Prot. Dosimetry 99, 283-286 (2002).

6. K. M. Prise, O. V. Belyakov, M. Folkard, A. Ozols, G. Schettino, B. Vojnovic and B. D. Michael, Investigating the cellular effects of isolated radiation tracks using microbeam techniques. Adv. Space Res. 30, 871-876 (2002).

7. S. Gerardi, A comparative review of charged particle microbeam facilities. Radiat. Prot. Dosimetry 122, 285-291 (2006).

8. M. Hada and A. G. Georgakilas, Formation of clustered DNA damage after high-LET irradiation: A review. J. Radiat. Res. 49, 203210 (2008).
9. S. V. Costes, A. Ponomarev, J. L. Chen, D. Nguyen, F. A. Cucinotta and M. H. Barcellos-Hoff, Image-based modeling reveals dynamic redistribution of DNA damage into nuclear sub-domains. PLoS Comput. Biol. 3, e155 (2007).

10. B. E. Nelms, R. S. Maser, J. F. MacKay, M. G. Lagally and J. H. Petrini, In situ visualization of DNA double-strand break repair in human fibroblasts. Science 280, 590-592 (1998).

11. B. E. Nelms, T. R. Mackie, J. F. MacKay, C. K. Hill, P. M. DeLuca, Jr., M. J. Lindstrom, J. Deasy and M. N. Gould, A comparison of cytotoxicity after whole- or partial-cell irradiation with synchrotronproduced ultrasoft X rays. Radiat. Res. 150, 521-527 (1998).

12. M. Folkard, G. Schettino, B. Vojnovic, S. Gilchrist, A. G. Michette, S. J. Pfauntsch, K. M. Prise and B. D. Michael, A focused ultrasoft $\mathrm{X}$-ray microbeam for targeting cells individually with submicrometer accuracy. Radiat. Res. 156, 796-804 (2001).

13. M. Sowa Resat and W. F. Morgan, Microbeam developments and applications: a low linear energy transfer perspective. Cancer Metastasis Rev. 23, 323-331 (2004).

14. C. T. Chantler, K. Olsen, R. A. Dragoset, J. Chang, A. R. Kishore, S. A. Kotochigova and D. S. Zucker, X-Ray Form Factor, Attenuation and Scattering Tables (v. 2.1). NIST, Gaithersburg, MD, 2005.

15. L. B. Schultz, N. H. Chehab, A. Malikzay and T. D. Halazonetis, 53BP1 is an early participant in the cellular response to DNA doublestrand breaks. J. Cell Biol. 151, 1381-1390 (2000).

16. I. Rappold, K. Iwabuchi, T. Date and J. Chen, Tumor suppressor p53 binding protein 1 (53BP1) is involved in DNA damage-signaling pathways. J. Cell Biol. 153, 613-620 (2001).

17. L. Anderson, C. Henderson and Y. Adachi, Phosphorylation and rapid relocalization of 53BP1 to nuclear foci upon DNA damage. Mol. Cell. Biol. 21, 1719-1729 (2001).

18. Y. Huyen, O. Zgheib, R. A. DiTullio, Jr., V. G. Gorgoulis, P. Zacharatos, T. J. Petty, E. A. Sheston, H. S. Mellert, E. S. Stavridi and T. D. Halazonetis, Methylated lysine 79 of histone H3 targets 53BP1 to DNA double-strand breaks. Nature 432, 406-411 (2004).

19. E. Reznikova, J. Mohr, M. Boerner, V. Nazmov and P. J. Jakobs, Soft X-ray lithography of high aspect ratio SU8 submicron structures. Microsyst. Technol. 14, 1683-1688 (2008).

20. R. A. Meldrum, S. W. Botchway, C. W. Wharton and G. J. Hirst, Nanoscale spatial induction of ultraviolet photoproducts in cellular DNA by three-photon near-infrared absorption. EMBO Rep. 4, 1144 1149 (2003).

21. L. Lan, S. Nakajima, Y. Oohata, M. Takao, S. Okano, M. Masutani, S. H. Wilson and A. Yasui, In situ analysis of repair processes for oxidative DNA damage in mammalian cells. Proc. Natl. Acad. Sci. USA 101, 13738-13743 (2004).

22. P. O. Mari, B. I. Florea, S. P. Persengiev, N. S. Verkaik, H. T. Bruggenwirth, M. Modesti, G. Giglia-Mari, K. Bezstarosti, J. A. Demmers and D. C. van Gent, Dynamic assembly of end-joining complexes requires interaction between Ku70/80 and XRCC4. Proc. Natl. Acad. Sci. USA 103, 18597-18602 (2006).

23. C. Dinant, M. de Jager, J. Essers, W. A. van Cappellen, R. Kanaar, A. B. Houtsmuller and W. Vermeulen, Activation of multiple DNA repair pathways by sub-nuclear damage induction methods. J. Cell. Sci. 120, 2731-2740 (2007).

24. G. Iliakis, S. Kurtzman, G. Pantelias and R. Okayasu, Mechanism of radiosensitization by halogenated pyrimidines: effect of BrdU on radiation induction of DNA and chromosome damage and its correlation with cell killing. Radiat. Res. 119, 286-304 (1989).

25. G. Iliakis, Y. Wang, G. E. Pantelias and L. Metzger, Mechanism of radiosensitization by halogenated pyrimidines: effect of BrdU on repair of DNA breaks, interphase chromatin breaks, and potentially lethal damage in plateau-phase CHO cells. Radiat. Res. 129, 202211 (1992).

26. P. R. Turner and W. A. Denny, The mutagenic properties of DNA minor-groove binding ligands. Mutat. Res. 355, 141-169 (1996).

27. C. M. White, O. Heidenreich, A. Nordheim and T. A. Beerman, Evaluation of the effectiveness of DNA-binding drugs to inhibit transcription using the c-fos serum response element as a target. Biochemistry 39, 12262-12273 (2000). 